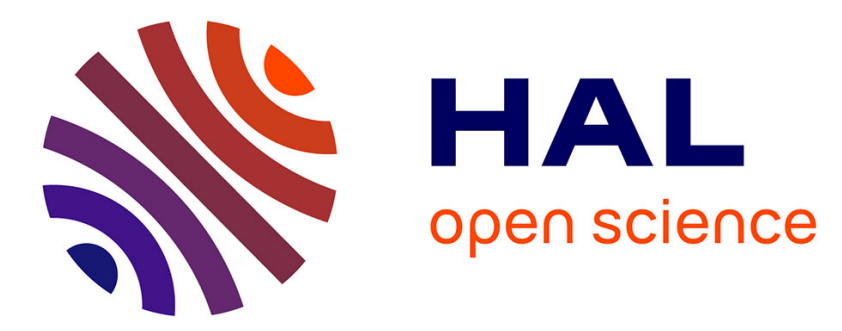

\title{
Cardiac motion assessement from echocardiographic image sequences by means of the structure multivector
}

Martino Alessandrini, Adrian Basarab, Hervé Liebgott, Olivier Bernard

\section{To cite this version:}

Martino Alessandrini, Adrian Basarab, Hervé Liebgott, Olivier Bernard. Cardiac motion assessement from echocardiographic image sequences by means of the structure multivector. IEEE International Ultrasonics Symposium - US 2013, Jul 2013, Prague, Czech Republic. pp. 1541-1544, 10.1109/ULTSYM.2013.0392 . hal-01147282

\section{HAL Id: hal-01147282 https://hal.science/hal-01147282}

Submitted on 30 Apr 2015

HAL is a multi-disciplinary open access archive for the deposit and dissemination of scientific research documents, whether they are published or not. The documents may come from teaching and research institutions in France or abroad, or from public or private research centers.
L'archive ouverte pluridisciplinaire HAL, est destinée au dépôt et à la diffusion de documents scientifiques de niveau recherche, publiés ou non, émanant des établissements d'enseignement et de recherche français ou étrangers, des laboratoires publics ou privés. 


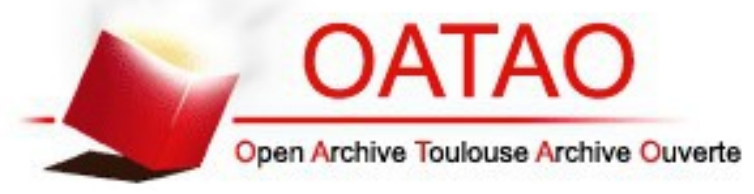

\section{Open Archive TOULOUSE Archive Ouverte (OATAO)}

OATAO is an open access repository that collects the work of Toulouse researchers and makes it freely available over the web where possible.

This is an author-deposited version published in : http://oatao.univ-toulouse.fr/ Eprints ID : 12454

To link to this article : DOI :10.1109/ULTSYM.2013.0392 URL : http://dx.doi.org/10.1109/ULTSYM.2013.0392

To cite this version : Alessandrini, Martino and Basarab, Adrian and Liebgott, Hervé and Bernard, Olivier Cardiac motion assessement from echocardiographic image sequences by means of the structure multivector. (2013) In: IEEE International Ultrasonics Symposium US 2013, 21 July 2013 - 25 July 2013 (Prague, Czech Republic).

Any correspondance concerning this service should be sent to the repository administrator: staff-oatao@,listes-diff.inp-toulouse.fr 


\title{
Cardiac motion assessment from echocardiographic image sequences by means of the structure multivector
}

\author{
Martino Alessandrini ${ }^{1}$, Adrian Basarab ${ }^{2}$, Hervé Liebgott, Olivier Bernard ${ }^{1}$ \\ ${ }^{1}$ Université de Lyon; CREATIS; CNRS UMR 5220; INSERM U1044; INSA-Lyon; Université Lyon 1; \\ ${ }^{2}$ Université Paul Sabatier Toulouse 3; IRIT UMR CNRS 5505;
}

\begin{abstract}
We recently contributed an algorithm for the estimation of cardiac deformation from echocardiographic image sequences based on the monogenic signal. By exploiting the phase information instead of the pixel intensity, the algorithm was robust to the temporal contrast variations normally encountered in cardiac ultrasound. In this paper we propose an improvement of that framework making use of an extension of the monogenic signal formalism, called structure multivector.

The structure multivector models the image as the superposition of two perpendicular waves with associated amplitude, phase and orientation. Such a model is well adapted to describe the granular pattern of the characteristic speckle noise. The displacement is computed by solving the optical flow equation jointly for the two image phases. A local affine model accounts for typical cardiac motions as contraction/expansion and shearing; a coarse-to-fine B-spline scheme allows for a robust and effective computation of the model parameters and a pyramidal refinement scheme helps deal with large motions. Performance was evaluated on realistic simulated cardiac ultrasound sequences and compared to our previous monogenic-based algorithm and classical speckle tracking. Endpoint-error was used as accuracy metric. With respect to them we achieved error reductions of $13 \%$ and $30 \%$ respectively.
\end{abstract}

Index Terms-echocardiography, motion estimation, image phase, structure multivector.

\section{INTRODUCTION}

Numerous techniques have been and are constantly proposed for the quantification of cardiac motion from echocardiographic image sequences and the derived mechanical quantities of deformation and strain have been proven reliable indicators for several pathologies as ischemia and dyssynchrony [1]. Among existing techniques, block matching, or speckle tracking, is to date the most consolidated one [1]. Block matching proceeds by finding the best match, as defined by the adopted dissimilarity measure, between two blocks extracted from two subsequent frames. Most common dissimilarity measures include cross-correlation, sum of absolute differences or sum of squared differences [1]. Alternative solutions belong instead to the main families of optical flow [2] and elastic registration [3].

Most of the existing solutions assume that the intensity of the region to track does not change over time (brightness constancy assumption). Nevertheless this assumption is rarely satisfied in cardiac ultrasound. The main reason is that the strength of the echo signal varies with the angle formed by the beam direction and the myocardial fibers, which changes in time due to heart motion. In this scenario phase-based methods have arisen a particular interest thanks to the property of the image phase of being strictly correlated with the image structure while insensitive to variations in the intensity profile. In particular the monogenic signal [4] has received a certain attention in the ultrasound community as a tool to compute the image phase. In particular our team recently described in [5] a novel motion estimation algorithm based on the monogenic phase that was shown to outperform a state-of-the art solution [2] exploiting brightness constancy.

Monogenic phase is computed by locally approximating the image as a 1D wave. Nevertheless, the 1D model is not ideal in the case of ultrasound images. Indeed, the typical granular texture determined by the speckle noise rather requires modelling structures whose size is limited in both dimensions. In this context more advanced image models than the monogenic signal exist that can be better adapted to the real structure of ultrasound images. In particular in this study we evaluate the feasibility of an alternative image processing tool called structure multivector [6].

In this new formalism the image is locally modelled as the superposition of two perpendicular waves with specific amplitude, phase and orientation. The displacement is computed by solving the optical flow equation jointly for the two image phases. A local affine model accounts for typical cardiac motions as contraction/expansion and shearing; a coarse-to-fine B-spline scheme allows for a robust and effective computation of the model parameters and a pyramidal refinement scheme helps deal with large motions. Performance was evaluated on realistic simulated cardiac ultrasound sequences and compared to our previous monogenic-based algorithm and classical speckle tracking.

The paper proceeds as follows. In Section II the motion estimation algorithm is presented. In Section III some implementation details are addressed. Results are presented in Section IV while conclusions are left to V.

\section{Proposed motion ESTIMATION ALGORITHM}

\section{A. The Structure Multivector}

The structure multivector [6] assumes the image $I(\mathbf{x})$, with $\mathbf{x}=[x, y]^{T}$ the pixel position, to be locally approximated by 


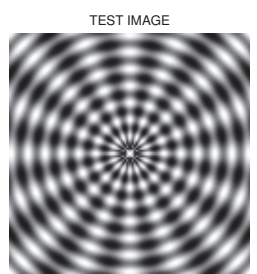

(a)

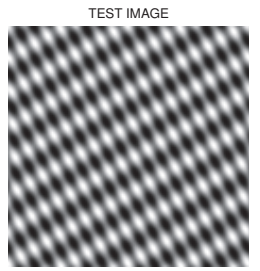

(d)

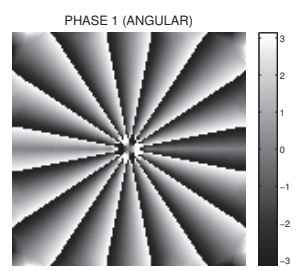

(b)

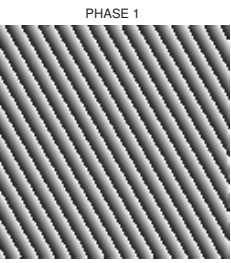

(e)

(f)
Fig. 1. First row from left to right: test image consisting of a superposition of an angular and a radial modulation, estimated first phase and estimated second phase. Second row from left to right: test image consisting of the superposition of two perpendicular sinusoids, estimated first phase and estimated second phase. Note how the two perpendicular components are locally well separated thanks to the structure multivector formalism.

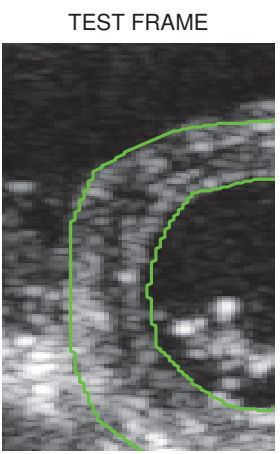

(a)
MONOGENIC

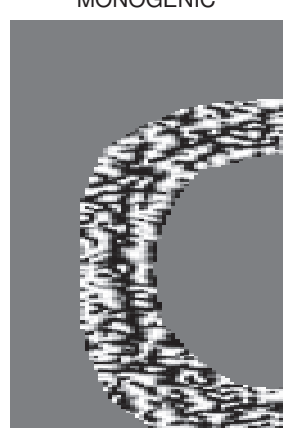

(b)

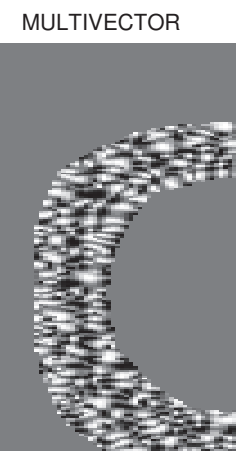

(c)
Fig. 2. (a) test ultrasound image representing the ventricular septum in a short axis view. (b) structure information retrieved with the monogenic signal, computed as $\cos \left(\varphi_{\text {monogenic }}\right)$. (c) structure information retrieved with the structure multivector, computed as $\cos \left(\varphi_{1}\right)+\cos \left(\varphi_{2}\right)$. Note how the 1D assumption simplifies the granular speckle texture, which is instead better taken into account by the structure multivector. Phase images has been reported inside the myocardium only for clarity sake.

two 1D signals $f_{1}$ and $f_{2}$ oriented in two orthogonal directions $\theta_{1}$ and $\theta_{2}=\theta_{1}+\pi / 2$, namely:

$$
I(\mathbf{x})=\sum_{i=1}^{2} I_{i}(\mathbf{x}) \quad \text { with } \quad I_{i}(\mathbf{x})=f_{i}\left(\mathbf{n}_{i}(\mathbf{x})^{T} \cdot \mathbf{x}\right),
$$

with $\mathbf{n}_{i}=\left[\cos \left(\theta_{i}(\mathbf{x})\right), \sin \left(\theta_{i}(\mathbf{x})\right)\right]^{T}$ the unit vector with direction $\theta_{i}$. Fig. 1(a) and (d) show examples of structures following the model in (1).

The two image components $I_{i}(\mathbf{x})$ can be separated from the responses to five $2 \mathrm{D}$ quadrature filters. The derivation of the filter set is not straightforward and won't be presented here for brevity sake. All relevant details are contained in the original paper [6]. Each component can be rewritten using the standard model of phase-based image processing:

$$
I_{i}(\mathbf{x})=\Re\left\{A_{i}(\mathbf{x}) \exp \left(i \varphi_{i}(\mathbf{x})\right)\right\}
$$

where local amplitude $A_{i}$ and local phase $\varphi_{i}$ has been introduced. The two amplitudes, phases and orientations represent the 6 features defining the adopted image model (1). The application of the structure multivector formalism on two test cases is illustrated in Fig. 1.

In the context of ultrasound the two-waves model provides a better description of the granular speckle texture as compared to the 1D model. This is illustrated in Fig. 2.

\section{B. Displacement Computation}

The displacement $\mathbf{d}=[u, v]^{T}$ is computed by assuming jointly conservation of the two image phases over time, namely $\varphi_{i}(\mathbf{x}, t+1)=\varphi_{i}(\mathbf{x}-\mathbf{d}(\mathbf{x}), t)$. Assuming small displacement the optical flow equations for the two phases are obtained:

$$
\begin{aligned}
& \varphi_{1 x} \cdot u+\varphi_{1 y} \cdot v+\varphi_{1 t}=0 \\
& \varphi_{2 x} \cdot u+\varphi_{2 y} \cdot v+\varphi_{2 t}=0
\end{aligned}
$$

where the notation $f_{x}=\partial_{x} f$ has been adopted. Although (3) is in principle solvable point-wise (two equations for two unknowns) the obtained result would be extremely sensitive to the presence of noise. The way around this issue is to solve (3) in the least-squares sense on a local window surrounding the pixel of interest, say $\mathbf{x}_{0}$. Assuming all pixels inside the local domain shift of the same quantity $\mathbf{d}_{0}$, the linear system of equations is hence obtained:

$$
\left\langle\mathbf{J}^{T} \mathbf{J}\right\rangle_{w} \cdot \mathbf{d}_{0}=-\left\langle\mathbf{J}^{T} \mathbf{b}\right\rangle_{w}
$$

with

$$
\mathbf{J}=\left(\begin{array}{cc}
\varphi_{1 x} & \varphi_{1 y} \\
\varphi_{2 x} & \varphi_{2 y}
\end{array}\right), \quad \mathbf{b}=\left(\begin{array}{c}
\varphi_{1 t} \\
\varphi_{2 t}
\end{array}\right)
$$

and $\langle\mathbf{v}\rangle_{w}$ the weighted average $\int_{\Omega} w\left(\mathbf{x}-\mathbf{x}_{0}\right) \mathbf{v}(\mathbf{x}) d \mathbf{x}$, where $w(\mathbf{x})$ the window function defining the local domain. Further details on the choice of $w$ will be given in the following. Note that unwrapping issues are never involved since phases appears only through their spatial and temporal derivatives. Temporal derivatives are easily computed by remembering that given two complex numbers the difference of their phases is equal to the phase of the product of the first with the conjugate of the second. Spatial derivatives are instead computed by exploiting the 1D assumption (1) which leads to $\nabla \varphi_{i}=\nu_{i} \cdot \mathbf{n}_{i}$, with $\nu_{i}=\mathbf{n}_{i}^{T} \cdot \nabla \varphi_{i}$ the local frequency and $\nabla=\left[\partial_{x}, \partial_{y}\right]^{T}$. Local frequency can be directly computed from the spatial derivatives of the filters involved in the structure multivector computation, similarly to [5], [6].

\section{Affine Model}

The assumption of pure translation within each block employed in (4) is too restrictive in the context of heart motion. A realistic model must indeed include also rotations, thickening/thinning and shear deformations normally observed in the myocardial tissue [2], [5]. These kind of deformations are naturally accounted for by an affine model. Considering 
a window $w$ centred at $\left(x_{0}, y_{0}\right)=(0,0)$, the affine model is written:

$$
\mathbf{d}(\mathbf{x})=\mathbf{A}(\mathbf{x}) \mathbf{u}, \quad \mathbf{A}=\left[\begin{array}{llllll}
1 & 0 & x & y & 0 & 0 \\
0 & 1 & 0 & 0 & x & y
\end{array}\right],
$$

where $\mathbf{u}=\left[d_{10}, d_{20}, d_{1 x}, d_{1 y}, d_{2 x}, d_{2 y}\right]^{T}$ is the new unknown vector: $d_{10}$ and $d_{20}$ correspond to the translation of the window center and $d_{i k}=\partial_{k} d_{i}$.

Plugging (6) into (4) leads to an underdetermined system of equations. The solution is then obtained by pre-multiplying both terms by $\mathbf{A}^{T}$, i.e. $\langle\mathbf{M}\rangle_{w} \mathbf{u}=\langle\mathbf{c}\rangle_{w}$, with $\mathbf{M}=\mathbf{A}^{T} \mathbf{J}^{T} \mathbf{J} \mathbf{A}$ and $\mathbf{c}=-\mathbf{A}^{T} \mathbf{J}^{T} \mathbf{b}$.

\section{Multiscale Choice of the Window Size}

The choice of the window size is a tedious issue connected with local techniques: the assumed motion model (translational or affine) may not hold when the window is too big, otherwise, the adoption of an excessively small window may result in the well known aperture problem. To circumvent this issue, in [2], [7] a multiscale strategy for locally choosing the most consistent window size was adopted. This is based on the possibility of computing the image moments, i.e., the entries of the system matrix $\mathbf{M}$ and the vector $\mathbf{b}$, at multiple scales, by using an efficient B-spline coarse-to-fine strategy. In particular, they are obtained from window functions $w$ that are progressively scaled and subsampled by a factor 2 in each dimension. More precisely, at scale $j$, the window $w^{j}\left(\mathbf{x}-\mathbf{x}_{0}\right)=w\left(\left(\mathbf{x}-2^{j} \mathbf{x}_{0}\right) / 2^{j}\right)$ is employed, where $w$ is written as the separable product of two B-spline functions. By doing so, at each scale $J_{f} \leq j \leq J_{c}\left(J_{f} \geq 0\right)$ a solution $\mathbf{u}^{j}$ can be computed. Among the scales considered, the $\mathbf{u}^{j}$ producing the smallest residual error $\left\|\mathbf{M u}^{j}-\mathbf{c}\right\|_{\ell 2} /|w|_{\ell 1}$ is retained as the final displacement estimate. Whenever necessary, bi-cubic interpolation is employed to obtain a dense motion field. With this strategy, the scale providing the most consistent motion estimate is selected.

\section{E. Iterative Displacement Refinement}

The hypothesis of small displacements employed in differential techniques may be inadequate whenever the displacement is substantial or the image intensity profile is non-linear. A possible way to deal with this limitation is to implement a form of Gauss-Newton optimization: the current estimate is used to undo the motion, and then the estimator is reapplied to the warped images to find the residual displacement [8]. When applied iteratively, this procedure can improve the estimation accuracy considerably. We employed the aforementioned refinement scheme in the algorithm presented.

\section{IMPLEMENTATION DETAILS}

The proposed algorithm was compared against block matching and our previous monogenic signal-based algorithm. The evaluation was performed on ultra-realistic synthetic 2D cardiac ultrasound sequences, generated according to the framework in [7] for which the ground-truth displacement was known. One short axis $(\mathrm{SaX})$ and one apical four chamber

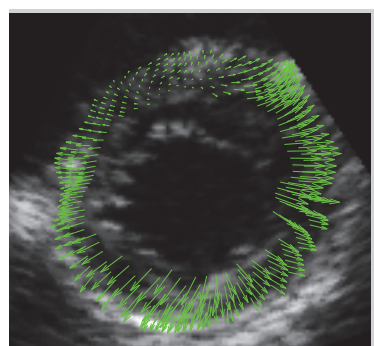

(a)

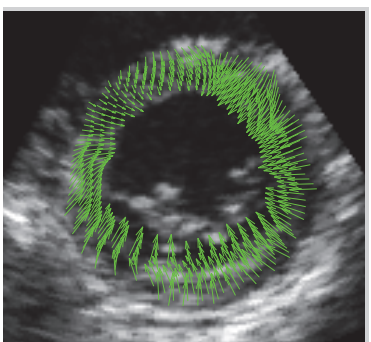

(b)
Fig. 3. (a),(b) Diastolic and systolic frames from a synthetic short axis sequence. Benchmark motion is superimposed as green arrows

(A4C) acquisitions were simulated. Image size was $179 \times 197$ pixels $^{2}$ and $271 \times 333$ pixels $^{2}$ for the $\mathrm{SaX}$ and A4C sequence respectively. The estimation accuracy was measured by means of the endpoint error (EE) defined as:

$$
E E(\mathbf{x})=\|\mathbf{d}(\mathbf{x})-\overline{\mathbf{d}}(\mathbf{x})\|_{2}
$$

where $\mathbf{d}$ and $\overline{\mathbf{d}}$ represent the estimated displacement and the ground truth respectively. Pixels belonging to the myocardium were considered in the evaluation only. Note that the displacement between subsequent frames is considered in this study. Examples frames from the SaX sequence are reported in Fig. 3 along with the benchmark displacement field.

Parameters for the three algorithms were optimized on the synthetic $\mathrm{SaX}$ sequence. In particular structure multivector was implemented as described in [6], with difference of Poisson (DoP) filter as lowpass kernel. The center wavelength $\lambda_{0}$ of the filter was set equal to 8 and divided by a factor 1.5 at each stage of the pyramidal refinement step. Four refinement iterations were employed. The multiscale window choice was implemented by considering fifth-order B-splines and scales $j=\{3,4,5\}$. Parameters of the monogenic based algorithm were set as in [5]. Block matching was implemented using rectangular blocks of size $8 \times 16$ pixels $^{2}$ with a search region of 2 pixels in both directions (consider maximum benchmark displacement was $<2$ pixels) and an interpolation factor of 10 (resolution $=1 / 10$ pixel) in both directions. Sum of absolute differences was used as dissimilarity metric. All algorithms were implemented in MATLAB (R2011b, The MathWorks, Natick, MA).

\section{RESULTS}

TABLE I

ESTIMATION ERRORS (MEAN VALUE \pm STANDARD DEVIATION)

\begin{tabular}{c|cc} 
Algorithm & SaX & A4C \\
\hline multivector (proposed) & $0.27 \pm 0.09$ & $0.23 \pm 0.06$ \\
monogenic [5] & $0.31 \pm 0.08$ & $0.26 \pm 0.05$ \\
block matching & $0.38 \pm 0.10$ & $0.34 \pm 0.08$ \\
\hline
\end{tabular}

The endpoint error evaluated globally (all pixels of all frames) on each simulated sequence for the three algorithms considered is reported in Table I. Both phase-based methods 


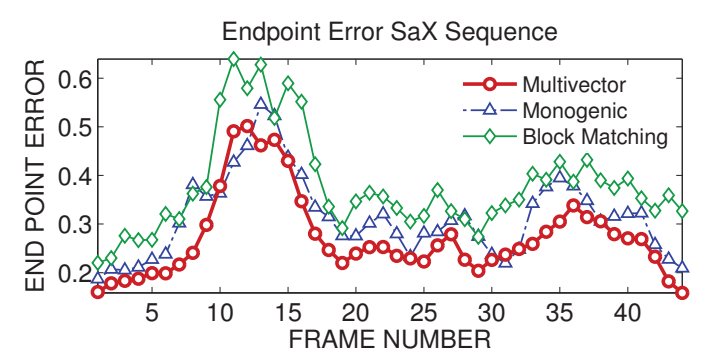

(a)

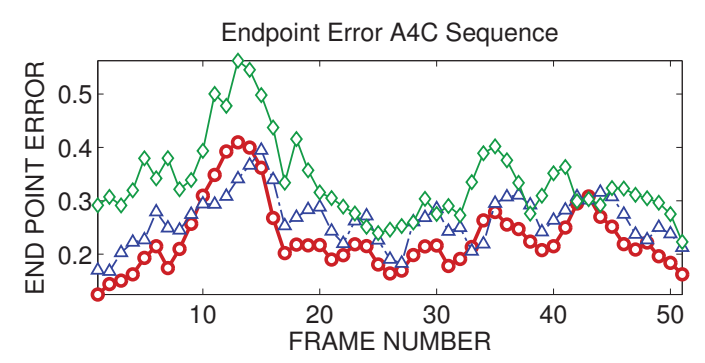

(b)

Fig. 4. (a),(b) Endpoint error for each frame of the SaX and A4C sequences.

outperform block matching in terms of accuracy. This is due to the fact that image phase is a more reliable feature as compared to pixel intensity as far as cardiac ultrasound in concerned, as confirmed by our previous findings [5], [9]. Moreover the proposed algorithm is the one returning the most precise velocity estimates. This is due to the fact that the $2 \mathrm{D}$ signal model assumed by the structure multivector represents a more accurate description of the ultrasound image as compared to the 1D model exploited by the monogenic signal.

The average endpoint error for each frame of the two simulated sequences is illustrated in Fig. 4. Note that the proposed algorithm is almost permanently the one returning the smallest estimation error, which confirms our previous observations. Overall, the relative error reduction with respect to the monogenic phase-based algorithm and block matching was of $13 \%$ and $30 \%$ respectively.

An ulterior advantage of the proposed framework with respect to block matching concerns the computational complexity. Indeed, while block matching implies interpolation to reach sub-pixel accuracy and then iteratively seeking within each block the shift returning the best match, in the proposed framework sub-pixel accuracy is directly obtained with no need of interpolation and the best shift is simply given by the solution of a linear system of equations, as explained in Section II. With the employed implementations, the speedup of both the considered phase-based solutions with respect to block matching was roughly $\times 50$.

\section{CONCLUSION}

Image phase has been proved a reliable feature for the estimation of cardiac motion with ultrasound. In this context this paper presented an improvement of a previous solution recently proposed by the authors based on the monogenic signal. The algorithm extracts two phase images from a single ultrasound frame and computes the displacement by assuming conservation of the two phases jointly over time. This decomposition is made possible thanks to a recent advanced image analysis tool called structure multivector. The proposed algorithm, evaluated on ultra realistic synthetic cardiac ultrasound sequences was shown to return more precise velocity estimates than the previous algorithm by the same authors and block matching.

Despite the structure multivector shows certain advantages over the monogenic signal, more flexible tools exist that could be better adapted to ultrasound images as the signal multivector [10], where the assumption of orthogonality between the two waves is relaxed. Investigating these tools will be the subject of future studies.

\section{ACKNOWLEDGEMENTS}

This work was partially supported by the US-Tagging grant financed by ANR (Agence Nationale de la Recherche). This work was performed within the framework of the LABEX Primes (ANR-11-LABX-0063) of Université de Lyon, within the program "Investissements d'Avenir" (ANR11-IDEX-0007) operated by the French National Research Agency (ANR).

\section{REFERENCES}

[1] J. D’hooge, A. Heimdal, F. Jamal, T. Kukulski, B. Bijnens, F. Rademakers, L. Hatle, P. Suetens, and G. R. Sutherland, "Regional Strain and Strain Rate Measurements by Cardiac Ultrasound: Principles, Implementation and Limitations," European Journal of Echocardiography, vol. 1, no. 3, pp. 154-170, Sep. 2000.

[2] M. Suhling, M. Arigovindan, C. Jansen, P. Hunziker, and M. Unser, "Myocardial motion analysis from b-mode echocardiograms," Image Processing, IEEE Transactions on, vol. 14, no. 4, pp. 525-536, 2005.

[3] A. Elen, C. Hon Fai, D. Loeckx, G. Hang, P. Claus, P. Suetens, F. Maes, and J. D'Hooge, "Three-dimensional cardiac strain estimation using spatio-temporal elastic registration of ultrasound images: A feasibility study," Medical Imaging, IEEE Transactions on, vol. 27, no. 11, pp. 1580-1591, 2008.

[4] M. Felsberg and G. Sommer, "The monogenic signal," Signal Processing, IEEE Transactions on, vol. 49, no. 12, pp. 3136-3144, 2001.

[5] M. Alessandrini, A. Basarab, H. Liebgott, and O. Bernard, "Myocardial motion estimation from medical images using the monogenic signal," Image Processing, IEEE Transactions on, vol. 22, no. 3, pp. 1084-1095, 2012.

[6] M. Felsberg and G. Sommer, "Image features based on a new approach to $2 \mathrm{~d}$ rotation invariant quadrature filters," in Computer Vision ECCV 2002, ser. Lecture Notes in Computer Science. Springer Berlin Heidelberg, 2002, vol. 2350, pp. 369-383.

[7] M. Alessandrini, H. Liebgott, D. Friboulet, and O. Bernard, "Simulation of realistic echocardiographic sequences for ground-truth validation of motion estimation," in Image Processing (ICIP), 2012 19th IEEE International Conference on, 30 2012-Oct. 3, pp. 2329-2332.

[8] J. R. Bergen, P. Anandan, K. J. Hanna, and R. Hingorani, "Hierarchical model-based motion estimation," in Computer Vision ECCV 1992, ser. Lecture Notes in Computer Science. Springer Berlin Heidelberg, 1992, vol. 588, pp. 237-252.

[9] M. Alessandrini, H. Liebgott, D. Friboulet, and O. Bernard, "Monogenic phase based myocardial motion analysis from cardiac ultrasound with transverse oscillations," in Ultrasonics Symposium (IUS), 2012 IEEE International, Oct., pp. 1098-1101.

[10] L. Wietzke and G. Sommer, "The signal multi-vector," Journal of Mathematical Imaging and Vision, vol. 37, pp. 132-150, 2010. 